\title{
On a Condition for the Nonsingularity of a Matrix
}

\author{
By Alston S. Householder
}

\begin{abstract}
A new derivation of a nonsingularity condition is given and related to conditions for the convergence of a matrix interation.
\end{abstract}

Gudkov [2] has recently published a condition, in the form of a system of inequalities, for the nonsingularity of a matrix, and Bailey and Crabtree [1] have extended this result by obtaining bounds for the determinant. They point out that the inequalities are consequences of a result due originally to Mehmke and Nekrasov, and further discussed by Ostrowski [5]. The original setting was in terms of the convergence of the Gauss-Seidel iteration. There might be some interest in returning to this original setting and rederiving these inequalities in somewhat more compact form.

The notation will be that used by the author in [3]. Let the matrix under consideration be

$$
A=D-B,
$$

where $D$ is null off the diagonal and $B$ is null on the diagonal. It is required that $D$ be nonsingular, whence there is no restriction in supposing $D=I$. Let $B$ be separated into its (proper) lower and upper components, whence

$$
A=I-L-R \text {. }
$$

It is known that

$$
\delta(I-|B|) \leqq \delta(I-B),
$$

whence there is no restriction in supposing $B \geqq 0$, and this will be done. If $H=$ $(I-L)^{-1} R$, Stein and Rosenberg ([6], see also [3]) have shown that one and only one of the three possibilities can hold:

$$
\rho(H)<\rho(B)<1, \quad \rho(H)=\rho(B)=1, \quad \rho(H)>\rho(B)>1 .
$$

In the first instance, both the Jacobi and the Gauss-Seidel iterations converge, otherwise neither converges. Hence, for $A$ to be nonsingular it is necessary and sufficient that $\rho(H)<1$. Evidently, $H \geqq 0$, whence $H e<e$ is a sufficient condition for this to hold. But these $n$ inequalities are those of Gudkov.

To see this, it is necessary first to state the Gudkov inequalities for this somewhat simplified form of the problem. They are

$$
\begin{aligned}
& \rho_{1}=\sum \rho_{1 i}<1, \\
& \rho_{i}=\sum \lambda_{i j} \rho_{i}+\sum \rho_{i j}<1, \quad i=2,3, \cdots, n .
\end{aligned}
$$

Received June 18, 1971.

AMS 1969 subject classifications. Primary 6535.

Key words and phrases. Matrices, nonsingularity, Gauss-Seidel interation.

Copyright @ 1972, American Mathematical Society 
It will be shown by an induction on the index $i$ that these inequalities are identical with the inequalities

$$
\mathrm{He}<\boldsymbol{e} .
$$

For $i=1$, it is obvious. Suppose it is verified for $i \leqq p$, and let $L_{1}$ represent the leading principal submatrix of $L$ of order $p$. Let $R_{1}$ likewise represent the leading principal submatrix of $R$ of order $p$, and let $\left(R_{1}, R_{2}\right)$ represent the first $p$ rows of $R$. Further, let $e_{1}$ and $e_{2}$ represent (deviating slightly from the notation in [3]), vectors of unit elements. Then, the hypothesis of the induction is that the first $p$ inequalities in (1) are representable by

$$
\left(I-L_{1}\right)^{-1}\left(R_{1} e_{1}+R_{2} e_{2}\right)<e_{1} .
$$

Now, adjoin a row and column to $I-L_{1}$, and a row to $\left(R_{1}, R_{2}\right)$. The first $p+1$ conditions in (2) are

$$
\left(\begin{array}{cc}
I-L_{1} & 0 \\
-l^{\prime} & 1
\end{array}\right)^{-1}\left(\begin{array}{cc}
R_{1} & R_{2} \\
0 & r^{\prime}
\end{array}\right)\left(\begin{array}{l}
e_{1} \\
e_{2}
\end{array}\right)<\left(\begin{array}{l}
e_{1} \\
1
\end{array}\right)
$$

Evidently,

$$
\left(\begin{array}{cc}
I-L_{1} & 0 \\
-l^{\prime} & 1
\end{array}\right)^{-1}=\left(\begin{array}{cc}
\left(I-L_{1}\right)^{-1} & 0 \\
l^{\prime}\left(I-L_{1}\right)^{-1} & 1
\end{array}\right),
$$

whence the condition that follows those in (3) is

$$
l^{\prime}\left(I-L_{1}\right)^{-1}\left(R_{1} e_{1}+R_{2} e_{2}\right)+r^{\prime} e_{2}<1 .
$$

But by the hypothesis of the induction, the first part of (4) coincides with the first summation on the right of (1), and the second part with the second summation. This completes the proof. To obtain from (1) the precise form used by Gudkov, one has only to introduce absolute value signs; multiply by $D$ and replace $\delta_{i} \rho_{i}$ by $\rho_{i}$; place $\delta_{i}$ on the right of the inequalities in (1); and modify the notation.

Department of Mathematics

University of Tennessee

Knoxville, Tennessee 37916

1. Duane W. Bailey \& Douglas E. Crabtree, "Bounds for determinants," Linear Algebra and Appl., v. 2, 1969, pp. 303-309. MR 41 \#6864.

2. V. V. GudKov, On a Certain Test For Non-Singularity of Matrices, Latvian Math. Yearbook 1965, "Zinatne", Riga, 1966, pp. 385-390. (Russian) MR 33 \#1323.

3. Alston S. Housenolder, The Theory of Matrices in Numerical Analysis, Blaisdell, Waltham, Mass., 1964. MR 30 \# 5475.

4. R. MEнMKE, K sposobu Zeǐdelja, služaščemu dlja rešenija sistemy lineǐnyh uravneniǐ s ves'ma bol'šym čislom neizvestnyh posredstvom posledovatel'nyh približenǐ̌. Izvlečenie iz pis'ma professoru Memke k professoru Nekrasovu (On Seidel's method for the solution of a system of linear equations with a large number of unknowns by means of approximations. From a letter from Professor Mehmke to Professor Nekrasov (in German).), Mat. Sb, v. 16, 1892, pp. 342-345.

5. Alexander M. Ostrowskr, "Determinanten mit überwiegender Hauptdiagonale und die absolute Konvergenz von linearen Iterationsprozessen," Comment. Math. Helv., v. 30, 1956, pp. 175-210. MR 17, 898.

6. P. STEIN \& R. L. Rosenberg, "On the solution of linear simultaneous equations by iteration," J. London Math. Soc., v. 23, 1948, pp. 111-118. MR 10, 485. 